\title{
Mutation of an RSV intronic element abolishes both U11/U12 snRNP binding and negative regulation of splicing
}

\author{
Richard R. Gontarek, Mark T. McNally, ${ }^{1}$ and Karen Beemon \\ Department of Biology, The Johns Hopkins University, Baltimore, Maryland 21218 USA
}

\begin{abstract}
A cis-acting negative regulator of splicing (NRS) within the gag gene of RSV is involved in control of the relative levels of spliced and unspliced viral mRNAs. Insertion of the NRS into the intron of an adenovirus pre-mRNA resulted in inhibition of splicing in vitro before the first cleavage step. Analyses of spliceosome assembly with this substrate showed that it formed large RNP complexes that did not migrate like mature spliceosomes on native gels. Affinity selection of the RNP complexes formed on NRS-containing pre-mRNAs showed an association with U11 and U12 snRNPs, as well as with the spliceosomal snRNPs.

Immunoprecipitation with antisera specific for U1 and U2 snRNPS showed binding of both snRNPs to NRS RNA. A 7-nucleotide missense mutation in the NRS that prevented binding of U11 and U12 snRNPs impaired NRS activity in vivo, suggesting a functional role for U11 and U12 snRNPS in the inhibition of splicing mediated by the RSV NRS RNA.
\end{abstract}

[Key Words: Retrovirus; RNA splicing; snRNPs]

Received June 23, 1993; revised version accepted July 28, 1993.

Retroviral replication involves reverse transcription of the RNA genome into DNA, which integrates into the host genome and is transcribed by cellular RNA polymerase II (for review, see Coffin 1990). While the viral RNA transcripts are processed, transported, and translated by cellular machinery, they yield both spliced and unspliced cytoplasmic mRNAs, unlike cellular premRNAs. The majority of the viral transcripts are not spliced but are transported to the cytoplasm, where they become full-length mRNA for Gag and Gag-Pol polyproteins, as well as packaged virion RNA. In the case of Rous sarcoma virus (RSV), the remainder of the primary transcripts are alternatively spliced in the nucleus to yield mRNAs for the env and src gene products. Production of infectious virus requires a balance of spliced and unspliced mRNAs.

Splicing of mRNA precursors occurs in a dynamic macromolecular complex called a spliceosome; the reaction requires ATP, functional splice site and branchpoint sequences, small nuclear ribonucleoproteins (snRNPs), heterogeneous ribonucleoproteins (hnRNPs), and essential non-snRNP splicing factors (for review, see Krainer and Maniatis 1988; Green 1991). The spliceosomal snRNP particles U1, U2, U4/U6, and U5 are abundant in animal cells $\left(10^{5}-10^{6}\right.$ copies per cell $)$ and participate in both steps of pre-mRNA splicing (for review, see Steitz et al. 1988). Genetic experiments have confirmed the sig-

${ }^{1}$ Present address: Department of Microbiology, Medical College of Wisconsin, Milwaukee, Wisconsin 53226 USA. nificance of RNA-RNA base-pairing between U1 snRNA and the 5' splice site (Zhuang and Weiner 1986) and U2 snRNA and the branchpoint (Wu and Manley 1989; Zhuang and Weiner 1989). The U4/U6 and U5 snRNPs, which appear to associate as a multi-snRNP complex (Konarska and Sharp 1987), have also been identified as components of the mature spliceosome (Frendewey and Keller 1985; Bindereif and Green 1987). Other less abundant snRNPs have been identified whose functions have not been defined (Kramer 1987; Reddy and Busch 1988). For example, Wassarman and Steitz (1992) have identified a complex containing U11 and U12 snRNPs and suggest that they probably function together in the cell, possibly in modulating specific RNA processing events.

Although the biochemistry of pre-mRNA splicing is now fairly well understood, less is known regarding the specificity of splice site selection and control of alternative splicing (for review, see Maniatis 1991). Several alternative mechanisms have been proposed to account for the accumulation of unspliced retroviral RNA; these involve either avoidance of the splicing machinery or a nonproductive interaction with the splicing pathway (Katz et al. 1988; Stoltzfus 1988; Coffin 1990). Both human immunodeficiency virus (HIV) and RSV have been found to have some suboptimal splice sites /Chang and Sharp 1989; Katz and Skalka 1990; Berberich and Stoltzfus 1991; Fu et al. 1991; McNally and Beemon 1992). In addition, negative regulatory sequences at a distance from splice sites have been identified in RSV that inhibit 
splicing in cis (Arrigo and Beemon 1988; Stoltzfus and Fogarty 1989; McNally et al. 1991). In the case of complex retroviruses such as HIV or human T-cell leukemia virus (HTLV), both viral-coded regulatory proteins (Rev or Rex) and cis-acting viral sequences are necessary for the productive cytoplasmic transport of unspliced or incompletely spliced viral mRNAs (for review, see Varmus 1988); however, most retroviruses, including RSV, do not encode similar regulatory proteins (Coffin 1990).

Previous work in our laboratory examined the effect of various deletions in an RSV proviral clone on the relative levels of spliced and unspliced viral RNAs in transient transfection assays (Arrigo and Beemon 1988). A 376nucleotide deletion from the gag gene of RSV results in increased levels of spliced RNA with a concomitant decrease in unspliced RNA. This cis-acting element is termed a negative regulator of splicing (NRS) because it appears to be necessary for the maintenance of wild-type levels of unspliced RNA (Arrigo and Beemon 1988). Similar effects of intronic deletions on the relative levels of RSV RNAs have been reported by Stoltzfus and Fogarty (1989). Insertion of the NRS into the introns of cellular pre-mRNAs causes them to accumulate as unspliced RNA when assayed in transfected cells (Arrigo and Beemon 1988; McNally et al. 1991). Deletion analysis has shown that a 151-nucleotide bipartite element is sufficient for NRS activity in vivo. The NRS was active only when in the sense orientation and within $\sim 300$ nucleotides of the 5' splice site (McNally et al. 1991).

The present study was undertaken to examine possible interactions between the NRS and nuclear factors in vitro. We present evidence that splicing of an adenovirus pre-mRNA substrate in vitro was inhibited by the presence of the NRS sequence in the intron. Furthermore, these NRS-containing pre-mRNAs did not form mature spliceosomes but sedimented in large RNP complexes. Substrates containing the NRS were associated with U11 and U12 snRNPs, as well as with the spliceosomal snRNPs. We provide evidence supporting a functional role for the observed U11/U12-NRS interaction, and we propose that binding of U11 and U12 snRNPs to this negative viral intronic element may interfere with assembly of functional spliceosomes.

\section{Results}

The NRS element inhibits splicing of an adenovirus pre-mRNA in vitro

Insertion of the NRS into the intron of a model premRNA derived from the adenovirus major late leader resulted in an increase in the ratio of unspliced/spliced RNA in transfected chicken embryo fibroblasts (CEFs) (McNally et al. 1991). This increase in the level of unspliced pre-mRNA occurred only with the NRS in the sense orientation, as was observed previously after insertion of the NRS into other heterologous introns (McNally et al. 1991). To test directly the effect of the NRS on splicing, we carried out in vitro splicing assays using similar adenovirus pre-mRNAs. A 151-nucleotide
NRS element containing RSV nucleotides 703-798 and 874-930 (BB $\Delta 76$, McNally et al. 1991) was inserted in both orientations into the intron of an SP6-driven transcription vector, $\mathrm{pAd} 2 \mathrm{H} / \mathrm{B}$, derived from the adenovirus 2 major late leader (Frendewey and Keller 1985). Radiolabeled pre-mRNAs (Fig. 1A) were then incubated in HeLa cell nuclear extracts under standard splicing conditions, and the products were monitored by denaturing gel electrophoresis on a $6 \%$ acrylamide gel (Fig. 1B).

After a 2-hr incubation in the HeLa nuclear extract, the parental Ad pre-mRNA yielded a 104-nucleotide spliced product and a 66-nucleotide first exon intermediate, as expected from accurate splicing (Fig. 1B, lane 4). The lariat intermediates derived from this substrate were visualized more readily on a $12 \%$ acrylamide gel (data not shown). In contrast, pre-mRNAs containing the NRS in the sense orientation, AdNRS $\mid+$ ), failed to generate any splicing intermediates or products after incubation for $2 \mathrm{hr}$ (Fig. 1B, lane 5). To test the sequence specificity of this splicing inhibition, we monitored the splicing of a substrate containing the NRS in the antisense orientation, AdNRS $\mid-1$. This pre-mRNA was spliced accurately, generating two lariat forms and a free first exon, as well as fully spliced mRNA (Fig. 1B, lane 6). Comparison of the rates of appearance of spliced $\mathrm{Ad}$ and AdNRS (-) mRNAs showed that the AdNRS(-) premRNA was spliced with reduced efficiency (data not shown). Previously, we have observed that large (376 nucleotides) NRS or nonspecific RNA insertions in the intron of Ad pre-mRNA inhibited splicing in vitro regardless of orientation (data not shown); thus, we attribute the reduction in splicing efficiency seen with AdNRS(-) to intron expansion. These results demonstrate that the NRS in the sense orientation is capable of inhibiting the splicing of a model precursor mRNA in vitro and suggest that splicing inhibition contributes to the accumulation of unspliced NRS-containing pre-mRNAs observed in vivo (Arrigo and Beemon 1988; McNally et al. 1991).

\section{Effect of the NRS on spliceosome assembly}

Because AdNRS + + transcripts failed to generate either spliced mRNAs or splicing intermediates (Fig. 1B), we concluded that splicing inhibition occurred before the first cleavage step. To characterize further the mechanism of NRS activity, we investigated spliceosome assembly with the NRS-containing pre-mRNA. The failure of these transcripts to splice could be the result of a block either before or after the formation of pre-spliceosomes or mature spliceosomes; alternatively, it could be the result of the formation of some aberrant splicing complex.

To determine whether spliceosome assembly was occurring on AdNRS $|+|$ pre-mRNAs, in vitro splicing reactions were analyzed first by electrophoresis on nondenaturing composite gels (Konarska and Sharp 1987; Zillman et al. 1988). The gel system that we used was shown previously by Zillman et al. (1988) to resolve three complexes: nonspecific complex I containing hnRNPs; prespliceosome complex A containing U1 and U2 snRNPs; 
Figure 1. The RSV NRS inhibits splicing of a model adenovirus pre-mRNA in vitro. $(A)$ Schematic representation of premRNA splicing substrates derived from the adenovirus major late leader. The stippled box denotes sense $(+\mid)$ or antisense (-) insertion of the RSV NRS fragment; bp denotes branchpoint adenosine; numbers refer to sizes (in nucleotides) of exon 1 , intron distance between $5^{\prime}$ splice site and insertion, NRS insert, intron distance between insert and $3^{\prime}$ splice site, and exon 2, respectively. $(B)$ Substrates described in $A$ were incubated in HeLa cell nuclear extract for $2 \mathrm{hr}$, and products were separated on a $6 \%$ acrylamide- $8 \mathrm{M}$ urea gel. The identities of the various intermediates and products are shown schematically. (Lanes 1-3) - ATP control reactions; (lanes 4-6) + ATP splicing reactions.
A

Ad

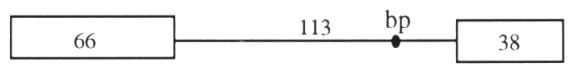

217

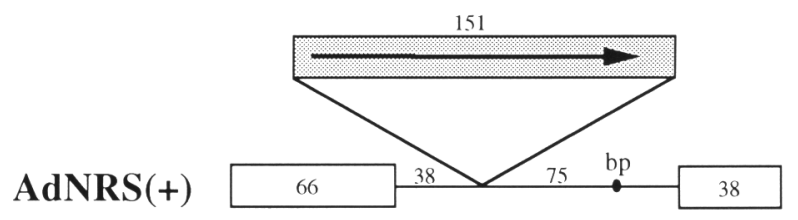

368

AdNRS(-)

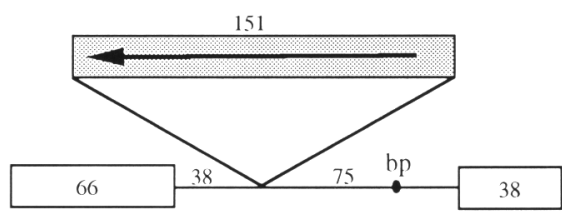

368 and spliceosome complex B containing U1, U2, and U4/ U6 snRNPs. Analysis of a splicing reaction containing the Ad pre-mRNA showed the stepwise formation of complexes I, A, and B. Pre-spliceosome A complexes were seen after $5 \mathrm{~min}$ of incubation (Fig. 2A, lane 2). After $30 \mathrm{~min}$, the Ad pre-mRNA formed large, slowly migrating B complexes indicative of spliceosome formation (lane 3). AdNRS (-) transcripts also formed three complexes (I, A, and B) that comigrated with those of the Ad pre-mRNA lacking the NRS (lane 7). However, the relative amount of the $B$ spliceosome complex on the AdNRS| - | pre-mRNA was lower than that on Ad. This was most likely attributable to expansion of the intron and correlated with the reduction in splicing efficiency observed previously with these substrates.

Formation of a complex comigrating with complex A on the AdNRS(+) pre-mRNA (Fig. 2A, lane 10) appeared similar to that on the Ad pre-mRNA (lane 2) after $5 \mathrm{~min}$ of incubation in the nuclear extract. However, after 30 min, the majority of NRS + +-containing transcripts still comigrated with complex A. Substantially less B complex (relative to A complex) formed with the AdNRS(+) pre-mRNA (lane 11) than with AdNRS(-) (lane 7) or Ad pre-mRNAs (lane 3). Analysis of Ad and AdNRS(-) premRNAs incubated in splicing reactions for $7 \mathrm{hr}$ showed that most of the complexes were no longer evident (lanes $4,8)$. However, the complexes containing the AdNRS(+) pre-mRNAs were unusually stable; a significant amount of complex comigrating with A remained after $7 \mathrm{hr}$ of incubation (lane 12). In summary, AdNRS $(+)$ premRNAs readily formed stable complexes that comigrated with presplicing complexes, but these did not mature into spliceosomes.

\section{NRS-containing pre-mRNAs form large RNP complexes}

To analyze further splicing complexes formed on NRScontaining substrates, we used sucrose gradient sedi-
B

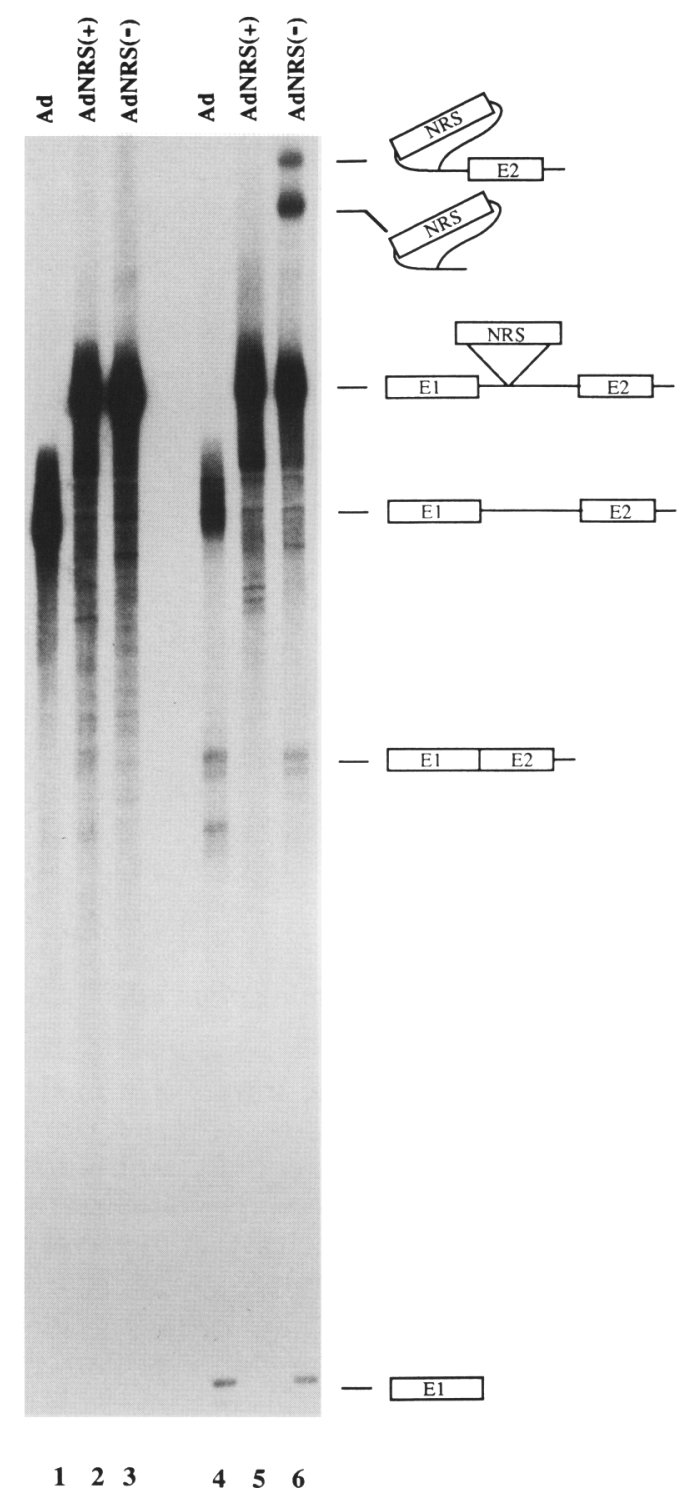


mentation. Analysis of in vitro splicing reactions by this method typically results in two distinct complexes: the pre-spliceosome and nonspecific complexes that sediment at $\sim 35 \mathrm{~S}$, and the functional spliceosome that sediments at 60S (Frendewey and Keller 1985; Grabowski et al. 1985). To detect differences in the sedimentation rates of complexes formed on different pre-mRNAs, we incubated ${ }^{35}$-labeled Ad and ${ }^{32}$ P-labeled AdNRS $|+|$ premRNAs separately under splicing conditions for $30 \mathrm{~min}$ and sedimented them together on a $10-30 \%$ sucrose gradient.

The Ad pre-mRNA was present in complexes that sedimented at both $35 \mathrm{~S}$ and $60 \mathrm{~S}$, as expected (Fig. 2B, open circles) (Frendewey and Keller 1985). Surprisingly, AdNRS| + | pre-mRNA was also found in two complexes, but both of these repeatedly sedimented more rapidly than their $35 \mathrm{~S}$ and $60 \mathrm{~S}$ Ad counterparts (Fig. 2B, solid circles). If the increased sedimentation rate resulted from the increased size of the AdNRS(+) transcript, AdNRS ( - ) transcripts would be expected to sediment more rapidly as well. However, the gradient-resolved splicing complexes formed on Ad and AdNRS(-) transcripts cosedimented (data not shown), ruling out the possibility that the shift observed with AdNRS $|+|$ was merely a result of increased transcript size. Thus, it appeared that the NRS sense sequences were responsible for the increased sedimentation rate.

\section{U1 and U2 snRNPs interact with NRS sequences}

The sequence of the NRS has several interesting features
(McNally et al. 1991), including three 5/9-nucleotide matches to the $5^{\prime}$ splice site consensus signal (nucleotides 702-710, 759-767 and 902-910) and an uninterrupted 15-nucleotide polypyrimidine tract upstream of a GAG (nucleotides 887-912) that is preceded by a sequence with 10/12-nucleotide homology to the rat calcitonin-specific branch sequence (nucleotides 875-886) (Adema et al. 1988; Emeson et al. 1989). Because these regions bear homology to $5^{\prime}$ and $3^{\prime}$ splice site and branchpoint consensus signals (Green 1991), we asked whether sequences within the NRS could bind U1 and U2 snRNPs. To address this, radiolabeled free NRS RNAs (not associated with Ad RNA) in both sense and antisense orientations, as well as Ad pre-mRNA, were transcribed in vitro and incubated in HeLa nuclear extracts for 30 min under splicing conditions. The reactions were immunoprecipitated using human antisera specific for U1 or U2 snRNPs (Mimori et al. 1984; Petterson et al. 1984); RNA was then extracted from the immune complexes and subjected to denaturing gel electrophoresis.

As expected, radiolabeled Ad pre-mRNA was immunoprecipitated with both $\alpha-\mathrm{U} 1$ and $\alpha-\mathrm{U} 2$ sera (Fig. 3, lanes 5,6). Sense RNA containing the NRS sequence (but no Ad sequences) was also immunoprecipitated with both sera (lanes 8,9 ), whereas antisense NRS RNA was not (lanes 11,12). The presence of NRS + | RNA in the immune complexes formed with both $\alpha-\mathrm{U} 1$ and $\alpha-\mathrm{U} 2$ sera suggested that $U 1$ and $U 2$ snRNPs interacted, either directly or indirectly, with NRS sequences. We propose that the binding of U1 and U2 snRNPs to the NRS sequences may affect the mobility of complexes formed on
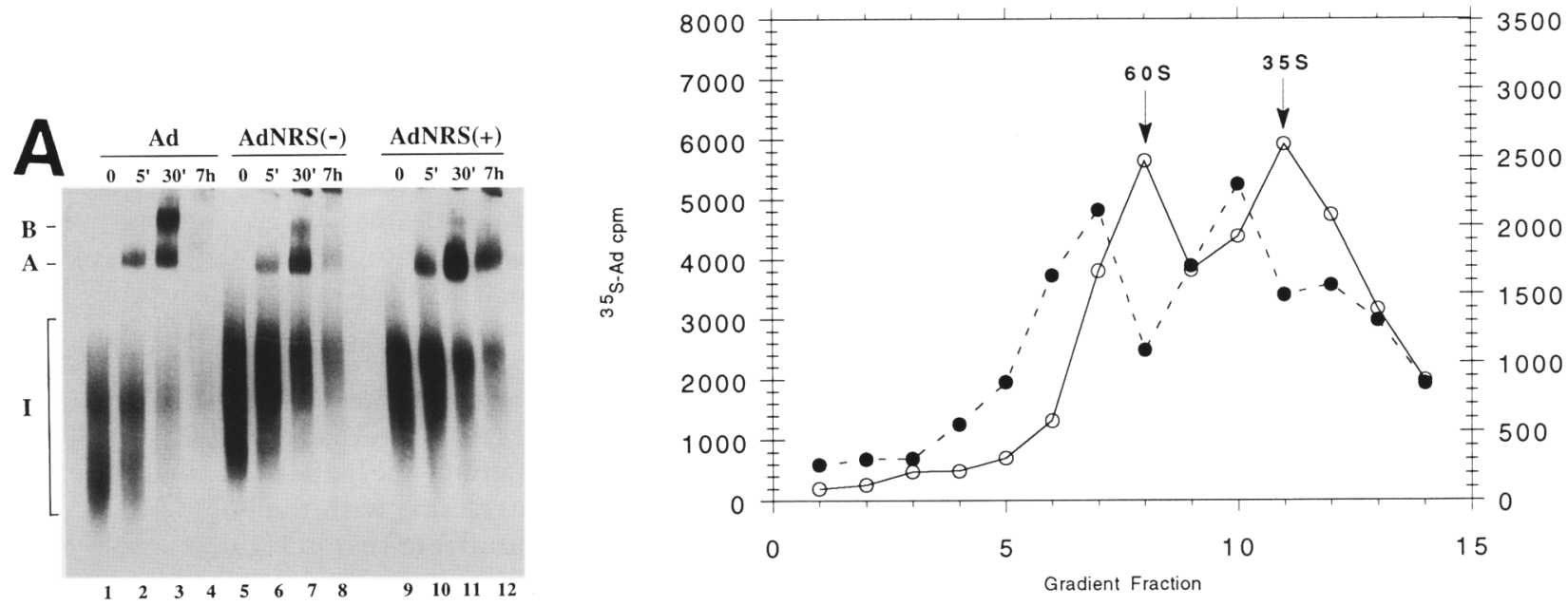

Figure 2. NRS-containing pre-mRNAs do not form mature splicing complexes but do form large RNP complexes. $(A)$ Native gel analysis of spliceosome assembly. Splicing reactions performed with Ad, AdNRS(-), and AdNRS(+) substrates were stopped at the indicated times and subsequently analyzed on native RNP gels. (I) Nonspecific complex; (A) pre-spliceosome complex; (B) mature spliceosome complex. $(B)$ Sucrose gradient analysis of spliceosome assembly. ${ }^{35}$ S-Labeled Ad pre-mRNA $(O)$ and ${ }^{32}$-labeled AdNRS( + | pre-mRNA / | were incubated in separate splicing reactions for $30 \mathrm{~min}$ and cosedimented through a $10-30 \%$ sucrose gradient. The positions of $35 \mathrm{~S}$ complexes and $60 \mathrm{~S}$ spliceosomes are indicated. 
NRS-containing RNA (Fig. 2B) and may play a role in the inhibition of splicing mediated by the NRS.

\section{Identification of factors present in large RNP complexes}

The composition of the large complexes formed on NRScontaining pre-mRNAs was investigated further by biotin-streptavidin affinity chromatography (Grabowski and Sharp 1986; Bindereif and Green 1987). Pre-mRNAs containing Biotin-11-UTP were incubated in HeLa nuclear extracts and selectively recovered on streptavidinagarose beads, together with associated factors. The RNA content of the selected complexes was assessed either by Northern analysis using snRNA-specific probes or directly by $3^{\prime}$ end-labeling. Because nonspecific binding of snRNPs to streptavidin-agarose has been demonstrated previously (Grabowski and Sharp 1986; Bindereif and Green 1987), parallel splicing reactions were performed on nonbiotinylated pre-mRNAs to measure the background.

An experiment using Northern analysis to assess the snRNA content of complexes bound to biotinylated and nonbiotinylated pre-mRNAs is shown in Figure 4. The complexes bound to the biotinylated Ad pre-mRNA (lane 5) contained U1, U2, U4, U5, and U6 snRNAs at levels above background (lane 2), indicative of spliceosome formation. U1 and U2 snRNAs were in greater relative abundance than $\mathrm{U} 4, \mathrm{U} 5$, and $\mathrm{U} 6$, suggesting that presplicing complexes were more abundant than spliceosomes in this reaction mixture. The same set of spliceosomal snRNAs was observed in association with the biotinylated AdNRS $\mid+$ | pre-mRNA (lane 3) and with AdNRS(-) pre-mRNA (lane 4). The results of this experiment suggested that transcripts containing the NRS bound the full set of spliceosomal snRNPs. Thus, it appeared that the failure of AdNRS $|+|$ pre-mRNAs to splice was not the result of the exclusion of spliceosomal snRNPs.

In an attempt to identify additional snRNPs that may be associating with the NRS, the biotinylated substrates were affinity selected as described above, and the selected snRNAs were then $3^{\prime}$ end-labeled with $\left[{ }^{32} \mathrm{P}\right] \mathrm{pCp}$ and RNA ligase and examined by denaturing gel electrophoresis. After 30 min of incubation, U1, U2, U4, and U5 snRNAs were associated with Ad and AdNRS $|+|$ premRNAs (Fig. 5, lanes 3,5) at levels above background (lane 6), confirming the result shown in Figure 4. We were not able to detect U6 snRNA in association with these substrates by $3^{\prime}$ end-labeling, presumably because its $3^{\prime}$ end is modified with a $2^{\prime}, 3^{\prime}$-cyclic phosphate that prevents efficient end labeling (Lund and Dahlberg 1991). Interestingly, an additional $3^{\prime}$ end-labeled band migrating slightly faster than U4 snRNA was observed in association only with AdNRS|+| pre-mRNA (band X, lanes 4,5 . The appearance of this small RNA species of $\sim 130$ nucleotides suggested that an additional snRNP might be interacting with the pre-mRNAs containing the NRS element.

Several minor snRNAs have been characterized (e.g.,

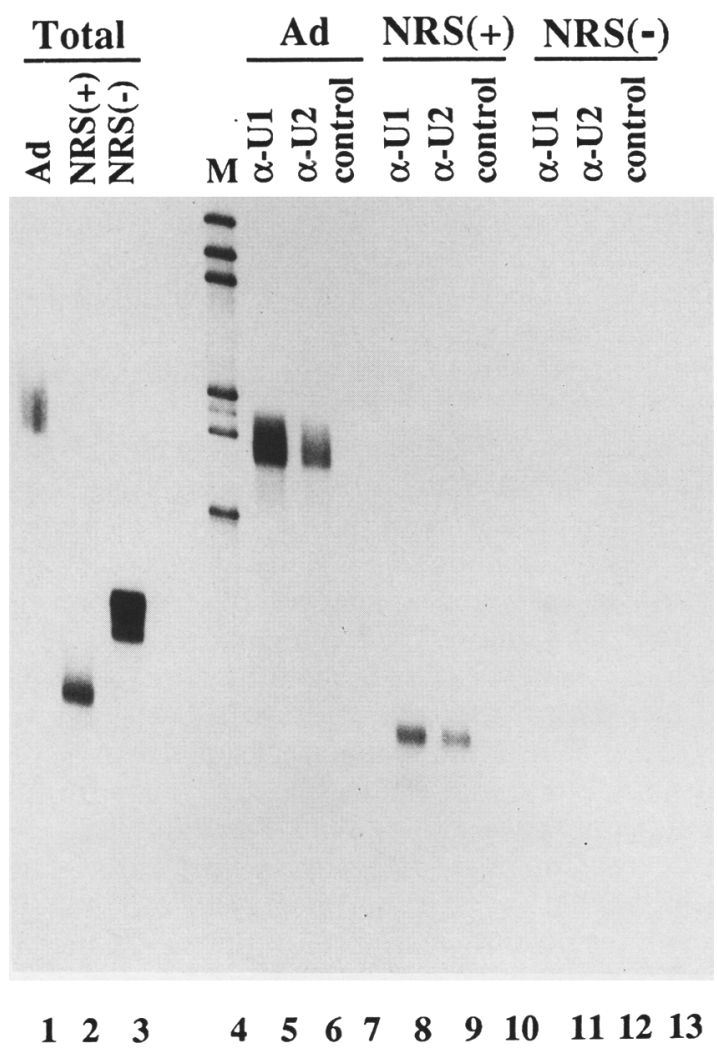

Figure 3. Human $\alpha$-U1 and $\alpha$-U2 antisera immunoprecipitate NRS RNA from nuclear extracts. Radiolabeled sense $(+)$ and antisense (- | NRS RNAs, as well as Ad pre-mRNA, were incubated in splicing reactions and directly immunoprecipitated with human antisera specific for U1 or U2 snRNPs $\mid \alpha-U 1$ and $\alpha$-U2) or a normal human serum (control). Lanes $1-3$ represent a portion of the total input substrates before immunoprecipitation; lane $4(M)$ is an RNA size marker (sizes in nucleotidess: $626,510,469,327,313,298$, and 252); lanes 5-13 show RNA extracted from immune complexes and electrophoresed on a $6 \%$ acrylamide- $8 \mathrm{M}$ urea gel.

U8, U9, and U11; Reddy and Busch 1988), of sizes that are consistent with the additional RNA species seen associated specifically with the AdNRS $(+\mid$ pre-mRNA (Fig. 5, lanes 4,5). Examination of the sequence and secondary structure of U11 snRNA (Montzka and Steitz 1988; Wassarman and Steitz 1992) showed a singlestranded region (loop 3) between nucleotides 63-71 that is complementary to two sequences near the $3^{\prime}$ end of the NRS (nucleotides 900-906 and 916-923). This observation led us to examine the possibility that the U11 snRNP might be interacting with the NRS.

A Northern blot similar to that in Figure 4 was hybridized with an RNA probe complementary to U11 snRNA (Wassarman and Steitz 1992). Because Wassarman and Steitz (1992) suggest that U11 and U12 snRNPs form a coparticle, the same blot was hybridized with a U12specific probe as well. We found that both U11 and U12 snRNAs were associated only with AdNRS $|+|$ transcripts (Fig. 6, lane 3). Because U12 snRNA comigrates 
with U1, it would have been difficult to detect by $3^{\prime}$ end-labeling (Fig. 5). Similar experiments carried out with biotinylated substrates containing NRS sequences alone also showed the binding of U11 and U12 snRNPs (data not shown), indicating that the binding sequence for these snRNPs in the AdNRS $|+|$ pre-mRNA was within the NRS element itself.

\section{A mutation that prevents binding of U11 and U12 snRNPs impairs NRS activity}

We then sought to determine the binding site (or sites) for U11 and U12 snRNPS within the NRS RNA. We observed two regions of the NRS (nucleotides 900-906 and 916-923) that were partially complementary to loop 3 of U11 snRNA. To determine whether these regions were necessary for U11 binding, we tested mutant NCPR lobtained from C. Cameron and J. Leis, Case Western Reserve University, Cleveland, $\mathrm{OH}$, which has $15 \mathrm{mis}-$ sense mutations in the NRS between nucleotides 902 and 922 , preventing potential base-pairing interactions at both sites (Fig. 7A). When the NCPR NRS mutant was

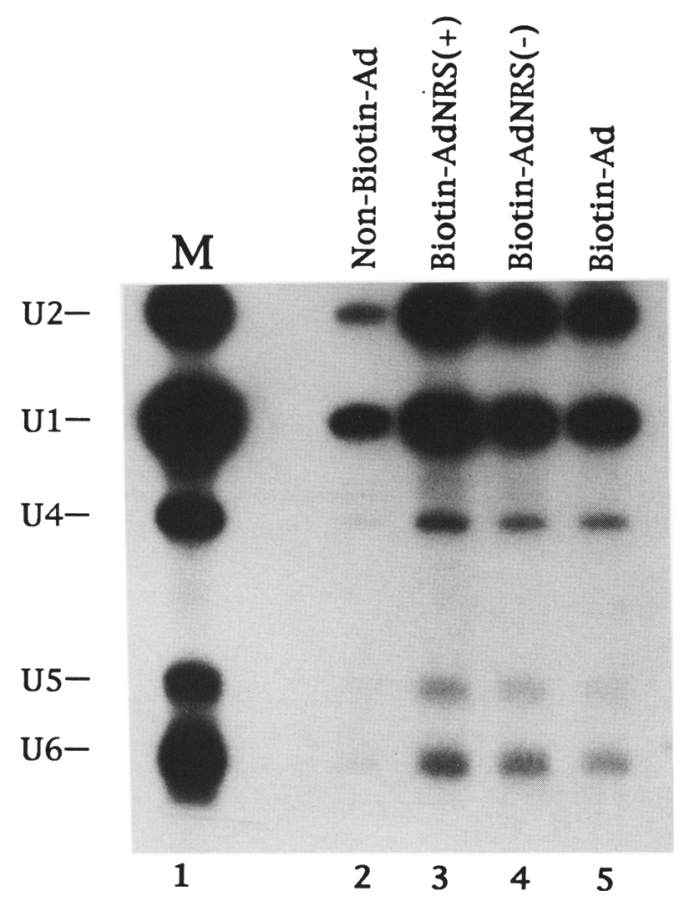

Figure 4. Northern blot analysis of snRNPs binding to premRNAs. Splicing reactions were carried out for $30 \mathrm{~min}$ on biotinylated or nonbiotinylated pre-mRNAs, followed by affinity selection on streptavidin-agarose. The bound snRNAs were eluted, electrophoresed on an $8 \%$ acrylamide- $8 \mathrm{M}$ urea gel, and electroblotted to a Zeta-probe nylon membrane. The membrane was probed with antisense RNA probes specific for U1, U2, U4, U5, and U6 snRNAs. Note that splicing reactions were performed on nonbiotinylated substrates to control for nonspecific binding of snRNPs to streptavidin-agarose. Three microliters of deproteinated HeLa nuclear extract was used as snRNA marker (M).

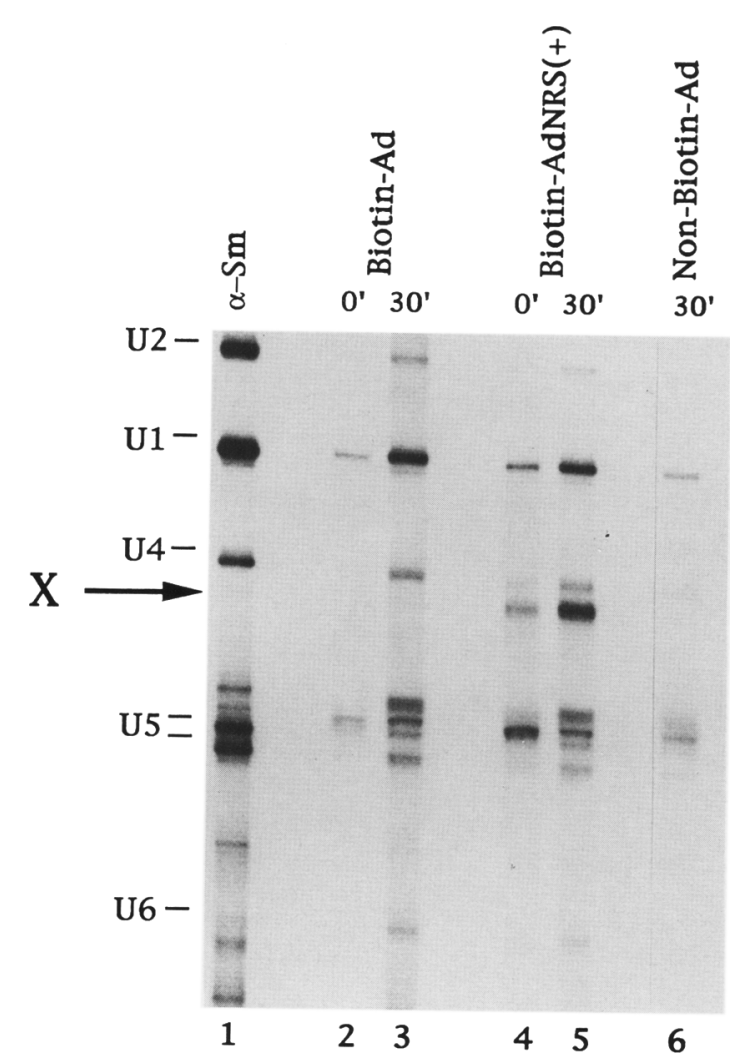

Figure 5. Affinity selection shows that an unidentified small RNA species associates with NRS-containing pre-mRNAs. Splicing reactions using biotinylated pre-mRNAs were performed for the indicated times and selected on streptavidinagarose as in Fig. 4. The eluted snRNAs were 3 '-end-labeled with $\left[{ }^{32} \mathrm{P}\right] \mathrm{pCp}$ and RNA ligase and separated on an $8 \%$ acrylamide- $8 \mathrm{M}$ urea gel. Lane 1 represents an $\alpha$-Sm immunoprecipitate of total HeLa nuclear extract that was 3 '-end-labeled to provide snRNA markers. Lane 6 is affinity-selected nonbiotinylated Ad pre-mRNAs used to control for background. Also indicated are 3 '-end labeled snRNAs. Band X represents an additional 3'-end-labeled small RNA species seen specifically associated with AdNRS| + | pre-mRNA.

inserted into the adenovirus intron, no binding of U11 or U12 was detected by affinity selection (Fig. 7B, lane 7), suggesting that nucleotides 902-922 were important for the binding of both snRNPS.

To localize further the U11 and U12 binding site (or sites) within this region, we then deleted the upstream potential binding site (nucleotides 900-906) from the NRS. However, this deleted RNA still bound U1l and U12 snRNPS (data not shown). The downstream potential binding site (nucleotides 916-923) was then altered with seven missense mutations to generate mutant RG11 (Fig. 7A). While RNA containing the wild-type NRS in the adenovirus intron bound U11 and U12 above background levels (Fig. 7B, lane 2), the NRS construct containing the RG11 substitution mutations did not (lane 3). Three other NRS fragments harboring substitutions upstream of nucleotides $916-923$ had no effect on U11/U12 binding (lanes 4-6). From this, we concluded 


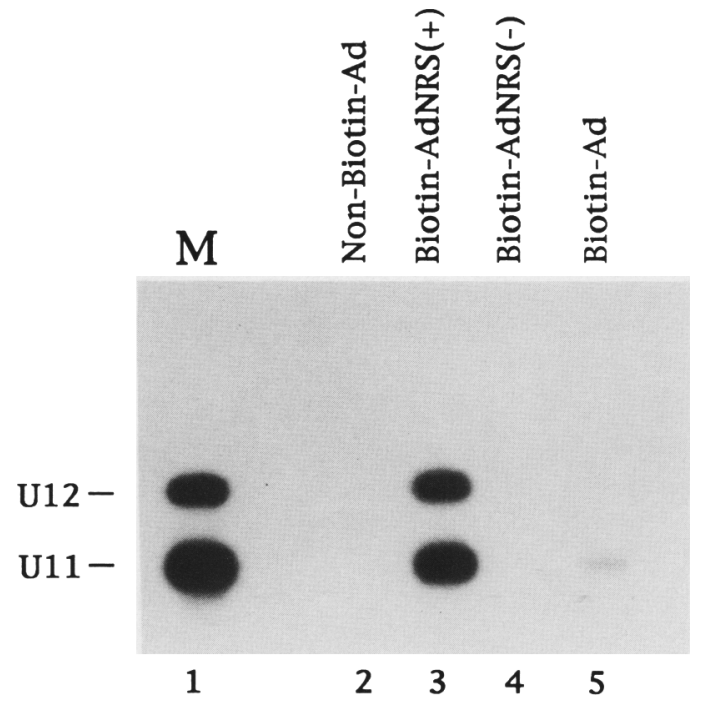

Figure 6. Affinity selection shows that U1l/U12 snRNPs bind to pre-mRNAs containing the NRS. A Northern blot similar to that shown in Fig. 4 was probed with a mixture of Ull and Ul2 antisense RNA probes. Three microliters of deproteinated HeLa nuclear extract was used as snRNA marker $(\mathrm{M})$.

A
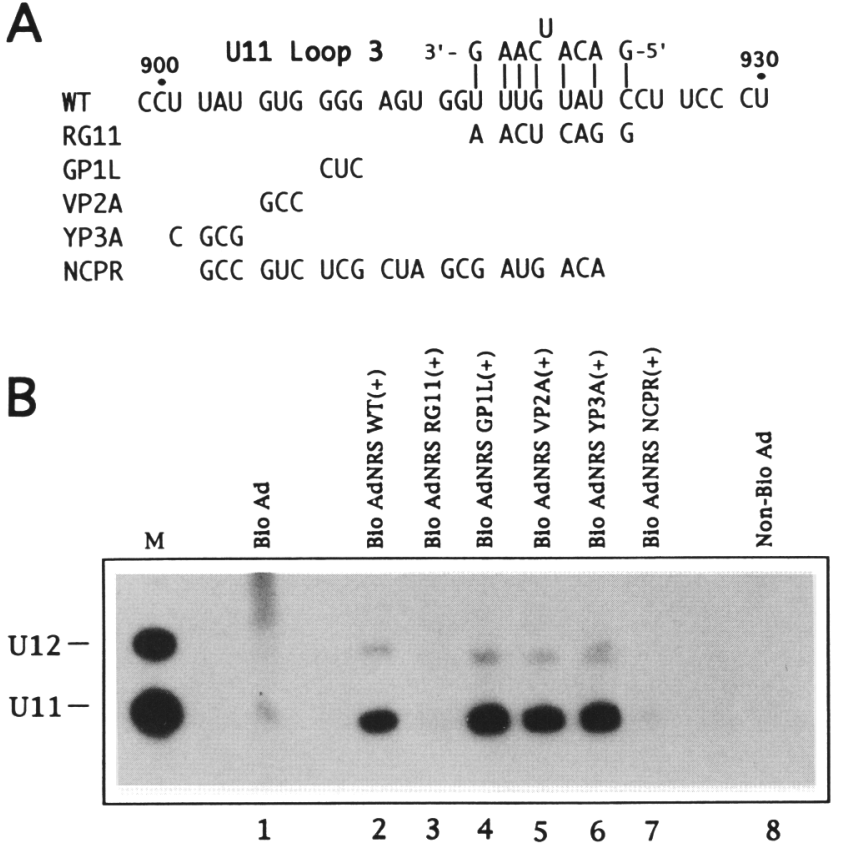

that nucleotides $916-923$ of the NRS are crucial for binding U11 and U12 snRNPs. Because this critical sequence is complementary to U11 snRNA, we propose that there is RNA-RNA base-pairing between the NRS and U11 snRNA at this site. Both NCPR and RG1l mutants also abolished binding of U12 snRNP.

We then asked whether a mutation that prevents binding of U11 and U12 snRNPs to the NRS in vitro would affect the ability of the NRS to increase the accumulation of unspliced RNA in vivo. Previously, we had shown that the NRS increased the ratio of unspliced/ spliced RNA in cells transfected with a heterologous test construct (Arrigo and Beemon 1988; McNally et al. 1991). Therefore, mutant and wild-type NRS fragments were inserted in both orientations into intron 2 of the chicken c-myc gene in pRSVNeo-int (Linial 1987), and the resulting constructs were transfected into CEFs. Total RNA was harvested after $48 \mathrm{hr}$ and subjected to RNase protection analysis using a probe designed to distinguish unspliced from spliced RNAs.

When the wild-type NRS fragment was inserted into the myc intron in the sense orientation (Fig. 7C, lane 2), the level of unspliced RNA increased to $56 \%$ of the total

Figure 7. A mutation that prevents binding of U1l and U12 snRNPs to the NRS in vitro severely impairs NRS activity in vivo. $(A)$ Nucleotide sequence of the $3^{\prime}$ end of the wild-type NRS element and of NRS mutants. A portion of loop 3 of U11 snRNA is also shown. $(B)$ Northern blot analysis of U11 and U12 snRNP binding to pre-mRNAs. Affinity selection and Northern blot analysis was carried out as described in Fig. 6 on Ad substrates containing wild-type and mutated NRS fragments. (C) Various NRS fragments were inserted in both orientations into pRSVNeo-int, and the resulting constructs were transfected into CEFs. RNA was harvested from transfected cells and subjected to RNase protection analysis using a probe designed to distinguish unspliced from spliced RNAs. (M) Markers used as RNA size standards (sizes in nucleotides: 626, 510, and 469). (Lane 1) Parental pRSVNeo-int; (lane 2-7) pRSVNeo-int containing the indicated NRS fragments in the sense $|+|$ ) and antisense $(-\mid$ orientations; (lane 8$)$ no DNA (mock transfection). Average percent unspliced RNA ( \pm S.D.) obtained from three independent experiments: myc, $14 \pm 7 \%$; NRS WT( $+1,56 \pm 7 \% ; \mathrm{NRS}$ WTi $-1,8 \pm 4 \%$; NRS RG $11+1,19 \pm 5 \%$; NRS RG1 $1-1,7 \pm 1 \%$; NRS YP3A $|+|, 50 \pm 4 \%$; and NRS YP3A $\mid-1,18 \pm 2 \%$. Below the autoradiograph is a schematic representation of the RNA generated from pRSVNeo-int, showing the position of the NRS insertions. Open boxes denote sequences from the Neo gene, solid boxes connected by the thin line denote myc gene exons and intron, and the stippled box represents SV40 sequences. The position of the riboprobe (which spans the myc 5' splice site) and the sizes of the protected fragments generated are also shown. 
compared with $14 \%$ unspliced RNA with the test construct lacking an insert (lane 1). When the mutated NRS fragment that failed to bind U11/U12 snRNPs was tested in the myc intron (NRS RG11, lane 4), levels of unspliced RNA fell to $19 \%$ of the total RNA. We observed similar results when mutant NCPR was tested (data not shown). Another mutation that did not affect $\mathrm{U} 11 / \mathrm{U} 12$ binding in vitro was tested here as well (NRS YP3A, lane 6) and resulted in similar levels of unspliced RNA as obtained with the wild-type NRS. These results suggest a functional role for binding of U11/U12 snRNPs in NRS-mediated splicing inhibition in vivo.

\section{Discussion}

Previous characterization of the RSV NRS element suggests that it is involved in maintaining the ratio of unspliced to spliced retroviral RNA in vivo /Arrigo and Beemon 1988; McNally et al. 1991; McNally and Beemon 1992). Here, we show that insertion of the NRS into the intron of an adenovirus pre-mRNA inhibited its splicing in vitro. Because the NRS RNA is capable of binding $U 1$, U2, U11, and U12 snRNPs, we propose that some snRNP-NRS interaction is involved in the block to mature spliceosome formation (Fig. 8). NRS missense mutants that blocked binding of U11 and U12 snRNPs exhibited diminished NRS activity in vivo, suggesting that U11/U12 binding is necessary for NRS-mediated splicing inhibition in RSV. Further testing of this model will include attempts to suppress the RG11 NRS mutant phenotype with U1l snRNA bearing compensatory mutations. Future work will also be directed toward understanding the significance of $U 1$ and $U 2$ binding to the NRS. It is possible that several distinct subpopulations of inhibitory NRS-snRNP complexes exist.

The affinity selection of U1l and U12 snRNPs by NRS-containing transcripts is intriguing, particularly because the cellular role of these minor snRNPs is unknown (Wassarman and Steitz 1992). Whereas U11 was initially shown to copurify with cleavage and polyade- nylation factor (CPF) (Christofori and Keller 1988), subsequent studies show that it is not required for polyadenylation (Takagaki et al. 1989; Bienroth et al. 1991). The association of U11 and U12 snRNPS with functional NRS elements suggests a possible regulatory role for these snRNPs in the inhibition of splicing mediated by the NRS. The limited abundance of Ull and U12 snRNAs in vivo $\left(10^{4}\right.$ and $5 \times 10^{3}$ copies per cell, respectively; Montzka and Steitz 1988/ may help to explain why a portion of the unspliced viral RNA is able to avoid the inhibitory influence of the NRS and splice faithfully in vivo.

Because NRS mutants that prevented binding of U11 snRNP altered an NRS sequence (nucleotides 916-923) complementary to Ull snRNA, we propose that there is RNA-RNA base-pairing between the NRS and U11 snRNA at this site. The same mutants also abolished binding of U12 snRNP. The interaction observed between U12 snRNP and the NRS could be the result of the binding of a U11/U12 complex (Wassarman and Steitz 1992). Alternatively, U12 may associate with prebound U11, or U12 may bind directly to the NRS at this same site. We have found that under high salt conditions, the amount of U12 binding to the NRS is often much less than that of U11 (e.g., see Fig. 7B), suggesting an unstable U11/ $\mathrm{U} 12$ complex. We did not detect any regions of significant complementary sequences between the NRS and U12. Thus, we favor the interpretation that the association between U12 and the NRS appears to be primarily mediated through U11-U12 interactions.

We have not observed functional splice sites within the NRS by either in vivo or in vitro assays (McNally et al. 1991). However, immunoprecipitation of free NRS RNA by $\alpha-U 1$ and $\alpha-U 2$ antisera indicated that sequences within the NRS were capable of binding U1 and U2 snRNPs and raises the possibility that these NRS sequences may be utilized as pseudosplice sites, perhaps interacting with genuine splice sites. Sequences that act as decoys or compete with authentic splicing signals have been identified in other systems. Siebel and Rio (1990) identified pseudo-5' splice sites flanking an au-

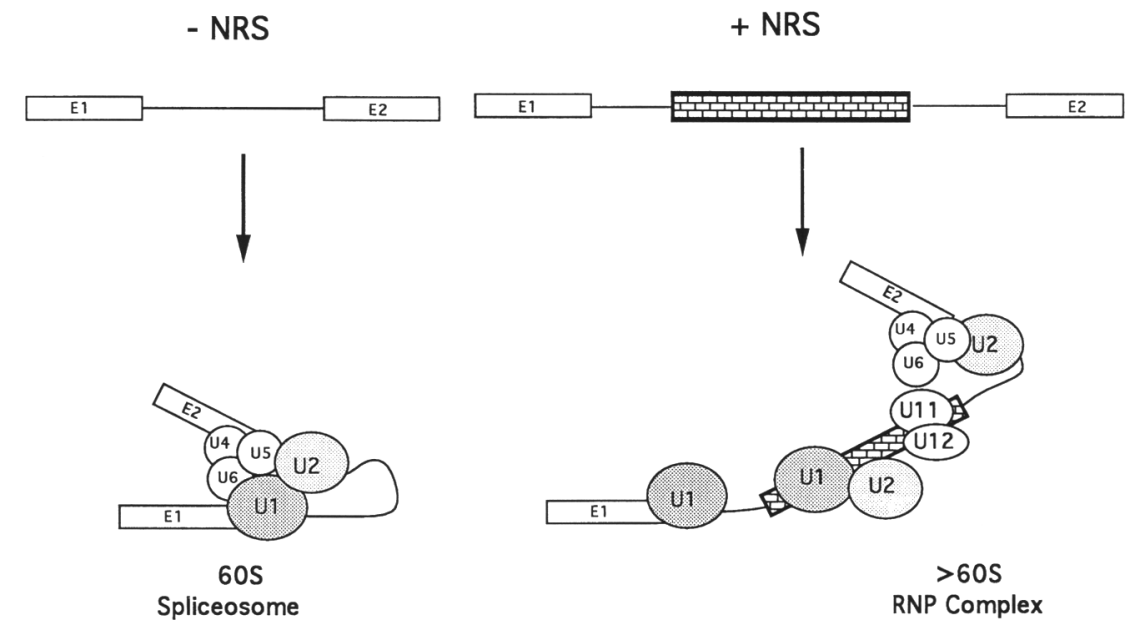

Figure 8. snRNP binding to the NRS may inhibit spliceosome assembly. Because the NRS RNA is capable of binding U1, U2, U11, and U12 snRNPs, we propose a model in which snRNP-NRS interactions are involved in the block to functional spliceosome formation. 
thentic $5^{\prime}$ splice site in the Drosophila P transposable element; competition between these sites appears to require their close proximity. However, in RSV viral RNA, the NRS is located in the intron, $\sim 300$ nucleotides from the $5^{\prime}$ splice site and 4000 nucleotides from the nearest 3' splice site (Schwartz et al. 1983). The large size of the minimal NRS element (151 nucleotides) (McNally et al. 1991) and the role of U11/U12 binding also argue against a simple splice site competition model.

One possible mechanism to account for the accumulation of unspliced retroviral RNA is the inaccessibility of the RNA to the splicing machinery (Stolzfus 1988; Coffin 1990). Fu et al. (1991) demonstrated that a truncated RSV pre-mRNA bearing the suboptimal env 3' splice site, but lacking the NRS, did not splice well in vitro and was not assembled efficiently into $60 \mathrm{~S}$ spliceosomes. Because spliceosome assembly was analyzed by sucrose gradient sedimentation in that study, it was not determined whether RSV RNA was efficiently associated with any splicing factors. In contrast, we found that the NRS-containing Ad transcripts did bind spliceosomal snRNPs and assembled large complexes that failed to splice. In the future, we plan to analyze RNP assembly using constructs containing both the env $3^{\prime}$ splice site and the NRS.

Although most unspliced RSV RNA is present in the cytoplasm, Arrigo and Beemon (1988) demonstrated that heterologous transcripts containing the NRS accumulate to a high level in the nucleus, making it unlikely that the NRS inhibits splicing through a rapid transport mechanism. The nuclear retention of unspliced NRS-containing RNAs may result from the stable association between the NRS and cellular factors observed in this study, if similar interactions are occurring in vivo. In support of this, $\alpha$-Sm antibodies that recognize the major snRNPs were found to be capable of precipitating NRScontaining unspliced RNA from transfected cell lysates (M. McNally and K. Beemon, unpubl.). The mechanism by which NRS-containing viral RNAs are transported to the cytoplasm remains unclear.

It has been shown recently that deleted RSV transcripts lacking the NRS element are not polyadenylated efficiently in vivo at the normal polyadenylation site in the viral LTR (Miller and Stoltzfus 1992). Previously, it has been suggested that splicing and polyadenylation of cellular mRNAs are linked in vivo /Villareal and White 1983), and polyadenylation of adenovirus RNAs in vitro is promoted by the presence of a $3^{\prime}$ splice site upstream of the poly(A) site (Niwa et al. 1990). Although the NRS is inhibitory to splicing, its association with snRNPs, including $\mathrm{U} 1, \mathrm{U} 2, \mathrm{U} 11$, and $\mathrm{U} 12$, may promote polyadenylation of unspliced retroviral RNAs at the weak LTR poly $(\mathrm{A})$ site.

\section{Materials and methods}

\section{Plasmid construction and synthesis of labeled RNA}

Viral DNA restriction fragments were from the Prague-C strain of RSV obtained from plasmid pATV-8K (Cobrinik et al. 1987), with the sequence coordinates determined by Schwartz et al. (1983). A BstNI fragment (nucleotides 703-930) from an RSV deletion mutant lacking nucleotides 799-874 (Carlberg et al. 1988) was end-repaired and cloned in both orientations into the unique BstEII site within the intron of $\mathrm{pAd} 2 \mathrm{H} / \mathrm{B}$ (Frendewey and Keller 1985) to yield pAdNRS $(+)$ and pAdNRS $(-)$. pAd2H/B contains exons 1 and 2 and the intervening intron of the adenovirus 2 major late transcriptional unit; both the intron and exon 2 have been truncated (Frendewey and Keller 1985). Capped, radiolabeled full-length pre-mRNAs were synthesized by SP6 transcription (Melton et al. 1984) of templates linearized with ScaI. End-repaired viral DNA fragments were also cloned into the SmaI site of pGEM-3Z, and labeled sense and anti-sense RNAs were synthesized by either T7 or SP6 transcription of templates linearized with either BamHI or EcoRI, respectively.

NRS mutant RG11 was generated by PCR amplification of a BstNI fragment from RSV with a $5^{\prime}$ primer corresponding to nucleotides 703-726 and a $3^{\prime}$ primer spanning nucleotides 907930 that changed nucleotides $916-923$ from TTTGTATC to AACTCAGG. Additional gag gene mutants involving the NRS element (NCPR, GP1L, VP2A, and YP3A) were kindly provided by C. Cameron and J. Leis. Mutant fragments were subcloned into $\mathrm{pAd} 2 \mathrm{H} / \mathrm{B}$, as described above, or into the unique SacII site of pRSVNeo-int (Linial 1987).

\section{In vitro splicing and gel electrophoresis}

HeLa cell nuclear extracts were prepared by the method of Dignam et al. (1983). In vitro splicing reactions were carried out essentially as described (Berget and Robberson 1986). RNA splicing reactions were analyzed on either $6 \%$ or $12 \%$ acrylamide- $8 \mathrm{M}$ urea gels as described (Frendewey and Keller 1985). For native gel analysis, splicing reactions were terminated by the addition of heparin and EDTA to $1.5 \mathrm{mg} / \mathrm{ml}$ and $10 \mathrm{~mm}$, respectively, and quick-frozen in a dry ice-ethanol bath. Samples were electrophoresed by the procedure of Zillman et al. (1988), using a 3.5\% polyacrylamide-bisacrylamide [80:1 (vol/ vol)] $-0.5 \%$ low melting temperature agarose composite gel run in $50 \mathrm{~mm}$ Tris-Cl, $50 \mathrm{~mm}$ glycine, and $10 \mathrm{~mm}$ EDTA (pH 8.8) at $15 \mathrm{~V} / \mathrm{cm}$ for $3-5 \mathrm{hrs}$ at $4^{\circ} \mathrm{C}$.

\section{Sucrose gradient analysis of splicing complexes}

One hundred-microliter splicing reactions were quick-frozen at $-80^{\circ} \mathrm{C}$, thawed at room temperature, and loaded onto 4-ml, $10-30 \%$ sucrose gradients containing $100 \mathrm{mM} \mathrm{KCl}, 1 \mathrm{~mm}$ $\mathrm{MgCl}_{2}$, and $10 \mathrm{mM}$ HEPES-KOH (pH 7.6) as described (Agris et al. 1989). Centrifugation was carried out in a Beckman SW60.1 Ti rotor for $1.5 \mathrm{hr}$ at $48,000 \mathrm{rpm}(310,000 \mathrm{~g})$. Equal volume fractions were collected from the bottom of each gradient and analyzed by scintillation counting. Escherichia coli ribosomal subunits were sedimented in a parallel gradient as markers.

\section{Immunoprecipitation}

Standard splicing reactions were subjected to direct immunoprecipitation as described in Zillman et al. (1987) using human antisera with specificity toward $\mathrm{U} 1$ and $\mathrm{U} 2$ snRNPs [anti(U1)RNP serum AG, and anti-(U2) serum Ya], a generous gift from Joan Steitz (Mimori et al. 1984; and Pettersson et al. 1984). Each of the labeled RNAs $\left(1 \times 10^{5} \mathrm{cpm}\right)$ was incubated under standard splicing conditions in a HeLa nuclear extract for 30 min. Five-microliter aliquots of the reaction were removed and incubated with $4 \mu \mathrm{l}$ of antiserum for $30 \mathrm{~min}$ on ice. Twenty microliters of prewashed Pansorbin (Calbiochem) was added and incubated on ice for $15 \mathrm{~min}$. Two hundred microliters of 
NET-M buffer $(0.05 \mathrm{M}$ Tris- $\mathrm{Cl}$ at $\mathrm{pH} 7.9,0.15 \mathrm{M} \mathrm{NaCl}, 0.05 \%$ NP-40, $0.5 \mathrm{~mm}$ DTT, $1.5 \mathrm{~mm} \mathrm{MgCl}_{2}$ ) was added, and immune complexes were pelleted for $15 \mathrm{sec}$ and washed three times in NET-M. Final pellets were resuspended in $0.1 \mathrm{M} \mathrm{NaCl}, 10 \mathrm{~mm}$ Tris-Cl (pH 8.0), 1 mM EDTA (pH 8.0), and $0.1 \%$ SDS, extracted once with an equal volume of phenol/chloroform-isoamyl alcohol $(24: 1)$, and ethanol precipitated in the presence of carrier glycogen. RNAs were analyzed on a $6 \%$ acrylamide $-8 \mathrm{~m}$ urea gel.

Biotin-streptavidin affinity chromatography, Northern blot analysis, and 3' end-labeling of snRNAs

Biotinylated RNAs were synthesized by including Biotin-11UTP (15\% of the total UTP, Enzo Biochemicals) in the standard transcription reaction. Biotinylated pre-mRNAs were incubated under splicing conditions and bound to streptavidin-agarose essentially as described (Bindereif and Green 1987), except that binding was carried out at $300 \mathrm{~mm} \mathrm{KCl}$. The snRNAs bound to biotinylated pre-mRNAs were eluted, $3^{\prime}$ end-labeled with 10 $\mu \mathrm{Ci}$ of $\left[{ }^{32} \mathrm{P}\right] \mathrm{pCp}(3000 \mathrm{Ci} / \mathrm{mmole}$, Amersham) and 5 units of RNA ligase (GIBCO BRL) (England et al. 1980), and electrophoresed on $8 \%$ polyacrylamide- $8 \mathrm{M}$ urea gels. Alternatively, specific snRNAs bound to biotinylated precursors were identified by affinity selection, electrophoresis on an $8 \%$ polyacrylamide- $8 \mathrm{M}$ urea gel, electroblotting to Zeta-probe nylon membrane (Bio-Rad), and probing with $10^{6} \mathrm{cpm} / \mathrm{ml}$ of U1, U2, U4, U5, and U6 RNA probes (Konarska and Sharp 1987) or U11 and U12 RNA probes (Wassarman and Steitz 1992).

\section{Analysis of NRS activity in vivo}

Transfection of pRSVNeo-int constructs containing the NRS into CEFs, isolation of total RNA, and RNase protection analysis were carried out as described previously (McNally et al. 1991). The products were electrophoresed on a $6 \%$ acrylamide- $8 \mathrm{M}$ urea gel. Gels were quantitated directly on a Molecular Dynamics PhosphorImager.

\section{Acknowledgments}

We thank Craig Cameron and Jonathan Leis for generously supplying RSV gag mutants prior to publication; Joan Steitz and Karen Wassarman for snRNP antibodies and plasmids; Victor Hou and Marguerite Ro for analysis of the NCPR mutant; Susan Berget, Martin Nemeroff, and Karen Wassarman for helpful discussions; and members of the Beemon laboratory for reviewing the manuscript. This work was supported by National Institutes of Health grant CA-48746. R.R.G. was supported by predoctoral National Research Service Award GM07231.

The publication costs of this article were defrayed in part by payment of page charges. This article must therefore be hereby marked "advertisement" in accordance with 18 USC section 1734 solely to indicate this fact.

\section{References}

Adema, G.J., R.A.L. Bovenberg, H.S. Jansz, and P.D. Baas. 1988. Unusual branch point selection involved in splicing of the alternatively processed, calcitonin/CGRP-1 pre-mRNA. $\mathrm{Nu}$ cleic Acids Res. 16: 9513-9526.

Agris, C.H., M.E. Nemeroff, and R.M. Krug. 1989. A block in mammalian splicing occurring after the formation of large complexes containing the U1, U2, U4, U5 and U6 small nuclear ribonucleoproteins. Mol. Cell. Biol. 9: 259-267.
Arrigo, S. and K. Beemon. 1988. Regulation of Rous sarcoma virus RNA splicing and stability. Mol. Cell. Biol. 8: 48584867.

Berberich, S.L. and C.M. Stoltzfus. 1991. Mutations in the regions of the Rous sarcoma virus 3' splice sites: Implications for the regulation of alternative splicing. J. Virol. 65: 26402646.

Berget, S.M. and B.L. Robberson. 1986. U1, U2, and U4/U6 small nuclear ribonucleoproteins are required for in vitro splicing but not polyadenylation. Cell 46: 691-696.

Bienroth, S., E. Wahle, C. Suter-Crazzolara, and W.J. Keller. 1991. Purification of the cleavage and polyadenylation factor involved in the $3^{\prime}$-end processing of messenger RNA precursors. I. Biol. Chem. 266: 19768-19776.

Bindereif, A. and M.R. Green. 1987. An ordered pathway of snRNP binding during mammalian splicing complex assembly. EMBO J. 6: 2415-2424.

Carlberg, K., T. Ryden, and K. Beemon. 1988. Localization and footprinting of an enhancer within the avian sarcoma virus genome. J. Virol. 6: 1617-1624.

Chang, D.D. and P.A. Sharp. 1989. Regulation by HIV-1 rev depends upon recognition of splice sites. Cell 59: 789-795.

Christofori, G. and W. Keller. 1988. 3' Cleavage and polyadenylation of mRNA precursors requires a Poly(A) polymerase, a cleavage factor, and a snRNP. Cell 54: 875-889.

Cobrinik, D., R. Katz, R. Terry, A.M. Skalka, and J. Leis. 1987. Avian sarcoma and leukosis virus pol-endonuclease recognition of the tandem long terminal repeat junction: Minimum site required for cleavage is also required for viral growth. $J$. Virol. 61: 1999-2008.

Coffin, J.M. 1990. Retroviridae and their replication. In Field's virology (ed. B.N. Fields and D.M. Knipe), pp. 1437-1500. Raven Press, New York.

Dignam, J.D., R.M. Lebovitz, and R.G. Roeder. 1983. Accurate transcription initiation by RNA polymerase II in a soluble extract from isolated mammalian nuclei. Nucleic Acids Res. 11: $1475-1489$.

Emeson, R.B., F. Hediran, J.M. Yeakley, J.W. Guise, and M.G. Rosenfeld. 1989. Alternative processing of calcitonin and CGRP mRNA is regulated at the calcitonin-specific splice acceptor. Nature 341: 76-80.

England, T.E., A.G. Bruce, and O.C. Uhlenbeck. 1980. Specific labeling of $3^{\prime}$ termini of RNA with T4 RNA ligase. Methods Enzymol. 65: 65-74.

Frendewey, D. and W. Keller. 1985. Stepwise assembly of a premRNA splicing complex requires U-snRNPs and specific intron sequences. Cell 42: 355-367.

Fu, X.-D., R.A. Katz, A.M. Skalka, and T. Maniatis. 1991. The role of branchpoint and $3^{\prime}$ exon sequences in the control of balanced splicing of avian retrovirus RNA. Genes \& Dev. 5: $211-220$.

Grabowski, P.J. and P.A. Sharp. 1986. Affinity chromatography of splicing complexes: U2, U5, and U4 + U6 small nuclear ribonucleoprotein particles in the spliceosome. Science 233: 1294-1299.

Grabowski, P.J., S.R. Seiler, and P.A. Sharp. 1985. A multicomponent complex is involved in the splicing of messenger RNA precursors. Cell 42: 345-353.

Green, M.R. 1991. Biochemical mechanisms of constitutive and regulated pre-mRNA splicing. Annu. Rev. Cell Biol. 7: 559599.

Katz, R.A. and A.M. Skalka. 1990. Control of retroviral RNA splicing through maintenance of suboptimal processing signals. Mol. Cell. Biol. 10: 696-704.

Katz, R.A., M. Kotler, and A.M. Skalka. 1988. cis-acting intron mutations that affect the efficiency of avian retroviral RNA 
splicing: Implications for mechanisms of control. I. Virol. 62: 2686-2695.

Konarska, M.M. and P.A. Sharp. 1987. Interactions between small nuclear ribonucleoprotein particles in the formation of spliceosomes. Cell 49: 763-774.

Krainer, A. and T. Maniatis. 1988. RNA splicing. In Frontiers in transcription and splicing (ed. B.D. Hames and D.M. Glover|, pp. 131-206. IRL Press, Oxford/Washington, D.C.

Kramer, A. 1987. Fractionation of HeLa cell nuclear extracts reveals minor small nuclear ribonucleoprotein particles. Proc. Natl. Acad. Sci. 84: 8408-8412.

Linial, M. 1987. Creation of a processed pseudogene by retroviral infection. Cell 49: 93-102.

Lund, E. and J.E. Dahlberg. 1991. 2', 3'-Cyclic phosphates and nontemplated nucleotides at the $3^{\prime}$ end of spliceosomal U6 small nuclear RNAs. Science 255: 327-330.

Maniatis, T. 1991. Mechanisms of alternative pre-mRNA splicing. Science 251: 33-34.

McNally, M.T. and K. Beemon. 1992. Intronic sequences and 3' splice sites control Rous sarcoma virus RNA splicing. $J$. Virol. 66: 6-11.

McNally, M.T., R.R. Gontarek, and K. Beemon. 1991. Characterization of Rous sarcoma virus intronic sequences that negatively regulate splicing. Virology 185: 99-108.

Melton, D.A., P.A. Krieg, M.R. Rebagliati, T. Maniatis, K. Zinn, and M.R. Green. 1984. Efficient in vitro synthesis of biologically active RNA and RNA hybridization probes from plasmids containing a bacteriophage SP6 promoter. Nucleic Acids Res. 12: 7035-7056.

Miller, J.T. and C.M. Stoltzfus. 1992. Two distant upstream regions containing cis-acting signals regulating splicing facilitate 3 '-end processing of avian sarcoma virus RNA. I. Virol. 66: 4242-4251.

Mimori, T., M. Hinterberger, I. Petterson, and J.A. Steitz. 1984. Autoantibodies to the $\mathrm{U} 2$ small nuclear ribonucleoprotein in a patient with scleroderma-polymyositis overlap syndrome. J. Biol. Chem. 259: 560-565.

Montzka, K.A. and J.A. Steitz. 1988. Additional low-abundance human small nuclear ribonucleoproteins: U11, U12, etc. Proc. Natl. Acad. Sci. 85: 8885-8889.

Niwa, M., S.D. Rose, and S.M. Berget. 1990. In vitro polyadenylation is stimulated by the presence of an upstream intron. Genes \& Dev. 4: 1552-1559.

Pettersson, I., M. Hinterberger, T. Mimori, E. Gottleib, and J.A. Steitz. 1984. The structure of mammalian small nuclear ribonucleoproteins. J. Biol. Chem. 259: 5907-5914.

Reddy, R. and H. Busch. 1988. Small nuclear RNAs: RNA sequences, structure, and modifications. In Structure and function of major and minor small nuclear ribonucleoprotein particles (ed. M.L. Birnstiel), pp. 1-37. Springer-Verlag, Berlin, Germany.

Schwartz, D.E., R. Tizard, and W. Gilbert. 1983. Nucleotide sequence of Rous sarcoma virus RNA. Cell 32: 853-869.

Siebel, C.W. and D.C. Rio. 1990. Regulated splicing of the Drosophila $\mathrm{P}$ transposable element third intron in vitro: Somatic repression. Science 248: 1200-1208.

Steitz, J.A., D.L. Black, K.A.Parker, A. Kramer, D. Frendewey, and W. Keller. 1988. Functions of the abundant U-snRNPs. In Structure and function of major and minor small nuclear ribonucleoprotein particles (ed. M.L. Birnstiel), pp. 115-154. Springer-Verlag, Berlin, Germany.

Stoltzfus, C.M. 1988. Synthesis and processing of avian sarcoma retrovirus RNA. Adv. Virus. Res. 35: 1-38.

Stoltzfus, C.M. and S.J. Fogarty. 1989. Multiple regions in the Rous sarcoma virus src gene intron act in cis to affect the accumulation of unspliced RNA. J. Virol. 63: 1669-1676.
Takagaki, Y., L. Ryner, and J.L. Manley. 1989. Four factors are required for 3 '-end cleavage of pre-mRNAs. Genes \& Dev. 3: 1711-1724.

Varmus, H. 1988. Regulation of HIV and HTLV gene expression. Genes \& Dev. 2: 1055-1062.

Villareal, L.P. and R.T. White. 1983. A splice junction deletion deficient in the transport of RNA does not polyadenylate nuclear RNA. Mol. Cell. Biol. 3: 1381-1388.

Wassarman, K. and J.A. Steitz. 1992. The low-abundance U11 and U12 small nuclear ribonucleoproteins (snRNPs) interact to form a two-snRNP complex. Mol. Cell Biol. 12: 12761285.

Wu, J. and J.L. Manley. 1989. Mammalian pre-mRNA branch site selection by U2 snRNP involves base pairing. Genes \& Dev. 3: 1553-1561.

Zhuang, Y. and A.M. Weiner. 1986. A compensatory base change in U1 snRNA suppresses a $5^{\prime}$ splice site mutation. Cell 46: 827-835.

- 1989. A compensatory base change in human U2 snRNA can suppress a branchpoint mutation. Genes \& Dev. 3: 1545-1552.

Zillman, M., S.D. Rose, and S.M. Berget. 1987. U1 small nuclear ribonucleoproteins are required early during spliceosome assembly. Mol. Cell. Biol. 7: 2877-2883.

Zillman, M., M.L. Zapp, and S.M. Berget. 1988. Gel electrophoretic isolation of splicing complexes containing U1 small nuclear ribonucleoprotein particles. Mol. Cell. Biol. 8: 814821 . 


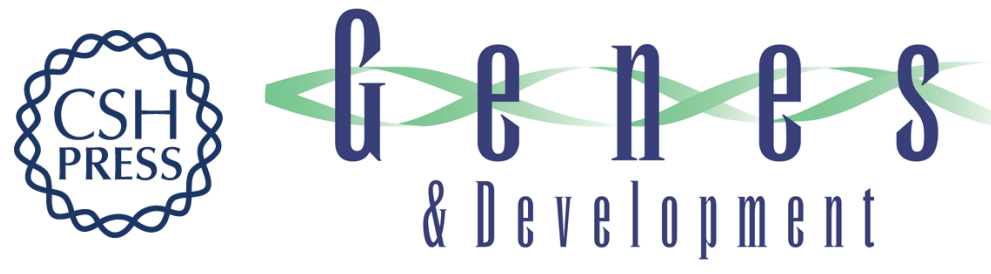

\section{Mutation of an RSV intronic element abolishes both U11/U12 snRNP binding and negative regulation of splicing.}

R R Gontarek, M T McNally and K Beemon

Genes Dev. 1993, 7:

Access the most recent version at doi:10.1101/gad.7.10.1926

References This article cites 47 articles, 27 of which can be accessed free at:

http://genesdev.cshlp.org/content/7/10/1926.full.html\#ref-list-1

License

Email Alerting

Service

Receive free email alerts when new articles cite this article - sign up in the box at the top right corner of the article or click here.

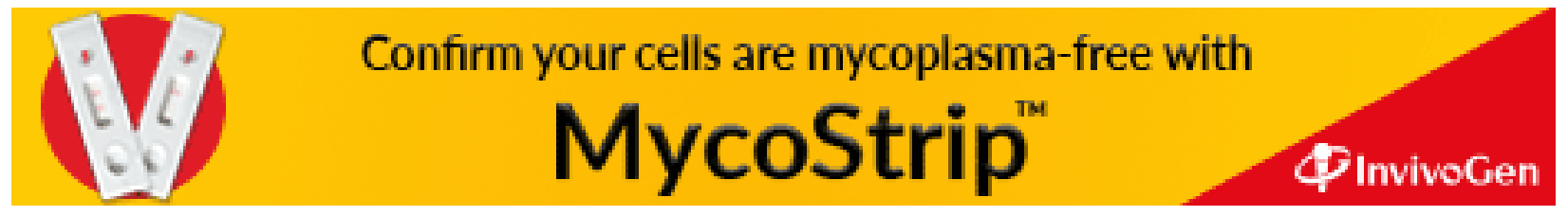

